\title{
The protective effect of hesperidin against renal ischemia-reperfusion injury involves the TLR-4/NF- $\kappa$ B/iNOS pathway in rats
}

\author{
X. MENG, M. WEI, D. WANG, X. QU, K. ZHANG, N. ZHANG and \\ XINJIAN LI*
}

Department of Nephrology, Affiliated Hospital of Jining Medical University, Jining, Shandong, 272001, PR China

Received: April 28, 2019 • Accepted: December 13, 2019

Published online: April 10, 2020

(c) 2020 Akadémiai Kiadó, Budapest

\begin{abstract}
Renal injury is reported to have a high mortality rate. Additionally, there are several limitations to current conventional treatments that are used to manage it. This study evaluated the protective effect of hesperidin against ischemia/reperfusion (I/R)-induced kidney injury in rats. Renal injury was induced by generating I/ $\mathrm{R}$ in kidney tissues. Rats were then treated with hesperidin at a dose of 10 or $20 \mathrm{mg} / \mathrm{kg}$ intravenously 1 day after surgery for a period of 14 days. The effect of hesperidin on renal function, serum mediators of inflammation, and levels of oxidative stress in renal tissues were observed in rat kidney tissues after I/Rinduced kidney injury. Moreover, protein expression and mRNA expression in kidney tissues were determined using Western blotting and RT-PCR. Hematoxylin and eosin (H\&E) staining was done for histopathological observation of kidney tissues. The data suggest that the levels of blood urea nitrogen (BUN) and creatinine in the serum of hesperidin-treated rats were lower than in the I/R group. Treatment with hesperidin also ameliorated the altered level of inflammatory mediators and oxidative stress in I/Rinduced renal-injured rats. The expression of $\mathrm{p}-\mathrm{I} \kappa \mathrm{B} \alpha$, caspase-3, NF- $\kappa \mathrm{B}$ p65, Toll-like receptor 4 (TLR-4) protein, TLR-4 mRNA, and inducible nitric oxide synthase (iNOS) was significantly reduced in the renal tissues of hesperidin-treated rats. Histopathological findings also revealed that treatment with hesperidin attenuated the renal injury in I/R kidney-injured rats. In conclusion, our results suggest that hesperidin protects against renal injury induced by $\mathrm{I} / \mathrm{R}$ by involving TLR-4/NF- $\kappa \mathrm{B} / \mathrm{iNOS}$ signaling.
\end{abstract}

\section{KEYWORDS}

hesperidin, kidney injury, ischemia/reperfusion, oxidative stress, inflammation

\footnotetext{
*Corresponding author. E-mail: 604479641@qq.com. Phone/Fax: +86 05372903672.
} 


\section{INTRODUCTION}

Acute renal injury (ARI) is a disorder with a high mortality rate; if a patient survives it, he/she is at increased risk of developing chronic renal failure [8]. In developing countries, $1 \%$ of total health expenditure costs are used for the treatment of diseases like ARI and its associated complications [2]. ARI patients are commonly reported to have decreased renal function, to suffer from ischemic disorders and sepsis, and to require renal transplantation [3]. Several factors are involved in the pathogenesis of ischemia/reperfusion (I/R)-induced renal injury. These include a dysfunction in energy metabolism, apoptosis, calcium overload, inflammatory mediators, and free radicals [12]. Conventional therapies available for the management of ARI have several limitations. In the last few decades, alternative medicine has proven to have a beneficial effect against the disease.

Molecules from natural sources are reported to be useful in the management of several disorders as alternative medicines. Hesperidin is a flavonoid that is isolated from plants belonging to the Lamiaceae and Betulaceae family [9]. Hesperidin has been shown to attenuate apoptosis, hypotension, oxidative stress, and inflammation [16, 21, 22]. It also protects against neuronal injury by ameliorating oxidative stress and inflammation [16]. Moreover, hesperidin has antimicrobial, anticancer, antiarthritic, anti-hypercholesterolemic, and anti-atherogenic activity $[1,10,14]$. Furthermore, hesperidin attenuates pancreatitis by inhibiting the TLR4/ IRAK1/NF- $\kappa$ B pathway [11]. Thus, this investigation evaluated the beneficial effect of hesperidin against $\mathrm{I} / \mathrm{R}$-induced $\mathrm{ARI}$ in rats.

\section{MATERIALS AND METHODS}

\section{Animals}

Male Sprague-Dawley rats that weighed 200-230 g each were purchased from Shanghai Medical College (Shanghai, China). Standard guidelines (humidity: $60 \pm 5 \%$; temperature: $24 \pm 3{ }^{\circ} \mathrm{C}$ ) were used to maintain the animals on a 12-h light/dark cycle. All study protocols were approved by the Institutional Animal Care and Use Committee of the Affiliated Hospital of Jining Medical University, Jining, China (IACUC/AH-JMU/2017-09).

\section{Chemicals}

Hesperidin was procured from Sigma Aldrich Ltd., USA and Biochemical estimation kits were purchased from Runyu Biotechnology Co., Shanghai, China. ELISA kits used for the determination of the level of inflammatory mediators and parameters of oxidative stress were procured from Runyu Biotechnology Co., Shanghai, China. Antibodies used for the western blot assay were procured from Abcam, Shanghai, China and Cell Signaling Technology, Denver, Colorado, USA.

Induction of ARI. All animals were anesthetized using an intraperitoneal injection of ketamine $(75 \mathrm{mg} / \mathrm{kg})$ and dexmedetomidine $(0.5 \mathrm{mg} / \mathrm{kg})$. Next, a surgical procedure was performed at a controlled temperature of $37 \pm 0.5^{\circ} \mathrm{C}$ by keeping the animal on a thermal heating pad. The left renal pedicle of the kidney was exposed by cutting the muscle and skin of the left abdomen. 
Renal pedicle vessels were then blocked for 45 min using an atraumatic bulldog clamp. Recovery of blood flow was achieved by removing the clamp. The animals were separated into four different groups: the control group (sham-operated), the I/R group (underwent I/R surgery and were treated with saline 1 day after surgery), and the hesperidin 10 and $20 \mathrm{mg} / \mathrm{kg}$ groups, which were treated intravenously with hesperidin at 10 or $20 \mathrm{mg} / \mathrm{kg}$, respectively, 1 day after surgery for a period of 14 days. Blood was then collected from the retroorbital plexus to examine biochemical parameters.

Assessment of renal function. Renal function was assessed by measuring the concentration of serum creatinine, total protein, and blood urea nitrogen (BUN) in the blood of all animals. The serum levels of creatinine, total protein, and BUN were estimated as per the manufacturer's protocol.

Assessment of inflammatory mediators and oxidative stress. Inflammatory mediators were measured using an ELISA assay. Inflammatory mediators including IL-10, IL-1 $\beta$, and TNF- $\alpha$ were estimated in the serum of I/R-induced renal-injured rats by using ELISA kits as per the manufacturer's protocol. The levels of malondialdehyde (MDA), reduced glutathione (GSH), xanthine oxidase (XO) activity, catalase (CAT), and superoxide dismutase (SOD) were also estimated in renal tissues as per the manufacturer's protocol. The level of inducible nitric oxide synthase (iNOS) in renal tissues was determined using an ELISA kit.

\section{Histopathology of renal tissues}

All animals were sacrificed and the kidneys of the animals were isolated. A $10 \%$ formalin solution was used to fix the kidney tissues, and paraffin was used to embed the tissues. A microtome was used to section the kidney tissues at a thickness of $4 \mu \mathrm{m}$, and the sections were stained using hematoxylin and eosin ( $\mathrm{H} \& \mathrm{E})$. A light microscope was used to analyze the sections. A score of $0-4$ was used to grade the percentage of necrosis and degeneration using the following parameters: normal $=0 ;<10 \%=1 ; 10-25 \%=2 ; 26-75 \%=3$; and $>75 \%=4$.

Western blotting. Kidney tissue homogenates were prepared and centrifuged at 12,000 RPM for $5 \mathrm{~min}$. A total protein extraction kit was used to extract protein from the tissue samples. A bicinchoninic acid assay was used to determine the concentration of protein. Next, $10 \%$ sodium dodecyl sulfate-polyacrylamide gel electrophoresis was used to separate the proteins, which were then transferred to a nitrocellulose membrane by electroblotting. Subsequently, the membrane was blocked with $5 \%$ non-fat milk and incubated in blocking buffer overnight at $4{ }^{\circ} \mathrm{C}$ with the following primary antibodies: anti-phosphorylated $\mathrm{I} \kappa \mathrm{B} \alpha$ (1:500 dilution), anti-caspase-3 (1:200 dilution), anti-NF- $\kappa$ B p65 (1:500 dilution), anti-TLR-4 (1:500 dilution), and anti- $\alpha$-tubulin (1:1000 dilution; loading control). Secondary goat antibodies conjugated to horseradish peroxidase (HRP) were added to the blocking buffer $(1: 1,000$; non-fat milk) and a chemiluminescence kit was used to detect proteins. A Bio-Rad detection system (Hercules, CA, USA) was used to record and visualize the immunoblots. Protein density was analyzed using ImageJ software (Bio-Rad, Shanghai, China).

$\boldsymbol{R T}$-PCR. RNA was isolated from separated spinal cord tissue with TRIzol Reagent (Thermo Fisher Scientific, Waltham, MA, USA). A RevertAid First Strand cDNA Synthesis Kit (Thermo 
Fisher Scientific) was used to reverse transcribe the RNA. The primers mentioned below were mixed with RT 2 SYBR Green Master Mix (Qiagen, Hilden, Germany) to analyze gene expression using Quantitative SYBR Green PCR assays (Applied Biosystems, Bedford, MA, USA). Primers used for the qRt-PCR were as follows: iNOS (forward: $5^{\prime}$-GTG GTG ACA AGC ACA TTT GG-3', reverse: $5^{\prime}$-GGC TGG ACT TTT CAC TCT GC-3'); TLR4 (forward 5' CCTCCCCTGTACCCTTCTCACTG-3', reverse $5^{\prime}$-ACGGCTACACCATTTTCCATTCC-3'); $\beta$-catenin (forward: $5^{\prime}$-GTCTTACCTGGACTCTGGAATCC-3', reverse: $5^{\prime}$-GGTATCCACATCCTCTTCCTCAG-3').

\section{RESULTS}

\section{Hesperidin ameliorates the markers of renal injury}

The effects of I/R-induced kidney injury in rats were confirmed (Fig. 1A and B). The effect of hesperidin was determined by estimating the serum concentrations of BUN and creatinine. The levels of BUN and creatinine were increased up to $63 \mathrm{mmol} / \mathrm{L}$ and $346 \mu \mathrm{mol} / \mathrm{L}$ in the serum of the control and I/R groups, respectively. However, treatment with hesperidin reduced the serum levels of creatinine $(156 \mu \mathrm{mol} / \mathrm{L})$ and BUN $(26 \mathrm{mmol} / \mathrm{L})$ compared to the I/R group.

\section{Hesperidin increases mediators of inflammation}

Inflammatory mediators in the serum of rats with I/R-induced kidney injury and hesperidin treatment were analyzed (Fig. 2). The levels of TNF- $\alpha$ (318 pg/mL), IL-1 $\beta$ (154 pg/mL), and IL$10(95 \mathrm{pg} / \mathrm{mL})$ were enhanced in the serum of the I/R group compared to the control group. There were lower levels of TNF- $\alpha$ and IL-1 $\beta$ (204 and $63 \mathrm{pg} / \mathrm{mL}$, respectively) in the serum of the hesperidin-treated group compared to the $\mathrm{I} / \mathrm{R}$ group of rats. Moreover, treatment with hesperidin increased the serum level of IL-10 (192 pg/mL) unlike in the I/R group.
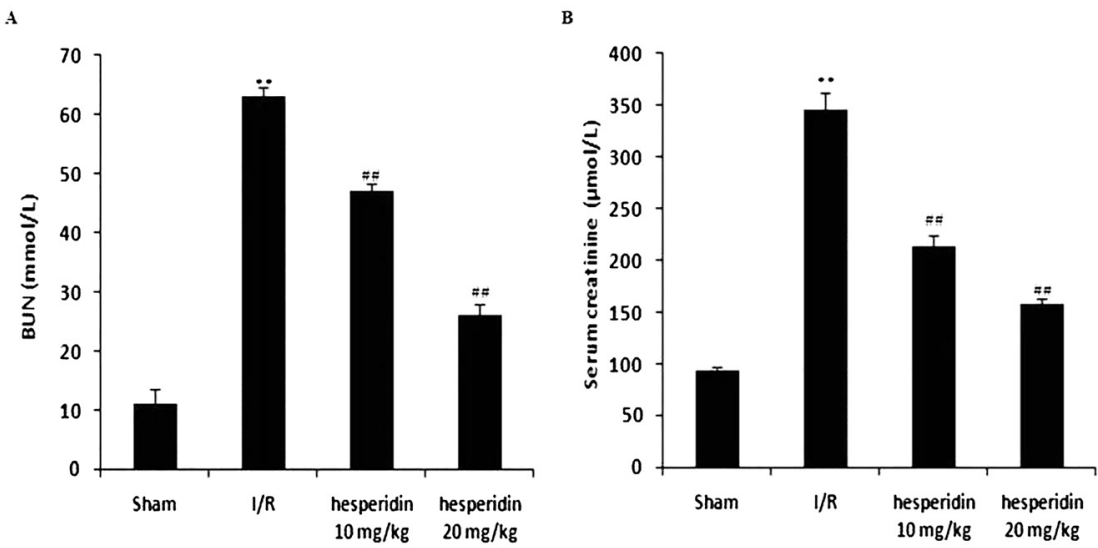

Figure 1. Hesperidin ameliorates the altered concentrations of markers involved in kidney injury caused by I/R. A: BUN. B: Creatinine. Data are presented as means \pm SEM $(n=10)$. ${ }^{* *} P<0.01$ compared to the control group; ${ }^{\# \#} P<0.01$ compared to the I/R group 


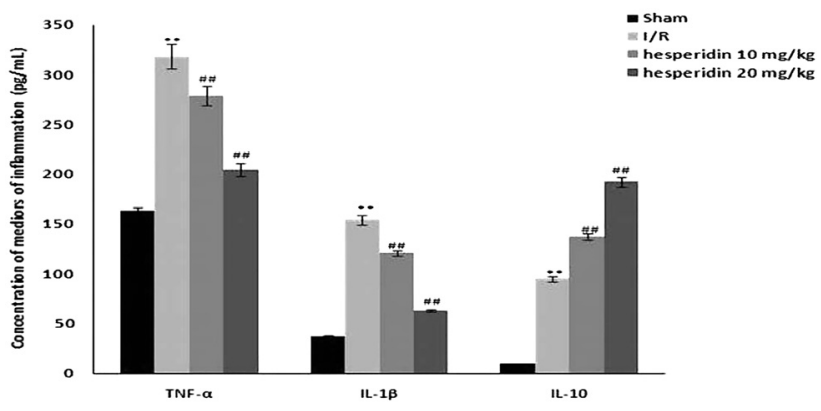

Figure 2. Hesperidin ameliorates the altered concentrations of inflammatory mediators in the serum of rats with I/R-induced kidney injury. Data are presented as means $\pm \operatorname{SEM}(n=10) .{ }^{* *} P<0.01$ compared to the control group; ${ }^{\# \#} P<0.01$ compared to the I/R group

\section{Hesperidin ameliorates parameters of oxidative stress}

The renal level of oxidative stress was determined by estimating parameters of oxidative stress in homogenates of kidney tissues from I/R-induced kidney-injured rats (Fig. 3A-F). There were significant $(P<0.01)$ increases in the levels of MDA, XO activity, and iNOS in the renal tissues of the I/R group compared to the control group. Moreover, the levels of GSH, SOD activity, and CAT were significantly $(P<0.01)$ reduced in the renal tissues of the I/R group compared to the control group. Treatment with hesperidin attenuated the altered parameters of oxidative stress in the renal tissues of rats with I/R-induced kidney injury.

\section{Hesperidin improves the histopathology of renal tissues}

The histopathology of renal tissues of rats with I/R-induced kidney injury was analyzed using H\&E staining (Fig. 4). Kidney tissues from control rats showed normal morphology. In contrast, kidney tissues from the I/R group showed leukocyte infiltration, swelling, congestion, hemorrhage, and tubules in the glomeruli. The kidney tissues from hesperidin-treated rats appeared to have normal morphology (Fig. 4A). The necrosis and degeneration scores were higher in the I/R group compared to the control group (Fig. 4B). There were significant reductions in the necrosis and degeneration scores in kidney tissues from hesperidin-treated rats compared to rats with I/R-induced kidney injury.

\section{Hesperidin ameliorates the expression of the TLR-4/NF- $\kappa$ B pathway}

In rats treated with hesperidin, the expression of Toll-like receptor 4 (TLR-4), NF- $\kappa \mathrm{B}, \mathrm{p}-\mathrm{I} \kappa \mathrm{B} \alpha$, and caspase-3 protein in renal tissues was analyzed (Fig. $5 \mathrm{~A}$ and B). The expression of TLR- 4 , NF- $\kappa \mathrm{B}$, $\mathrm{p}-\mathrm{I} \kappa \mathrm{B} \alpha$, and caspase- 3 protein was higher in the renal tissues of rats with $\mathrm{I} / \mathrm{R}$-induced kidney injury compared to the control group. However, rats treated with hesperidin showed a decrease in the expression of TLR $-4, \mathrm{NF}-\kappa \mathrm{B}, \mathrm{p}-\mathrm{I} \kappa \mathrm{B} \alpha$, and caspase- 3 protein compared to the I/R group.

\section{Hesperidin increases the mRNA expression of iNOS and TLR-4}

The effect of hesperidin on the TLR-4/iNOS signaling pathway was investigated by measuring the mRNA expression of TLR- 4 and iNOS in the renal tissues of rats with I/R-induced kidney 

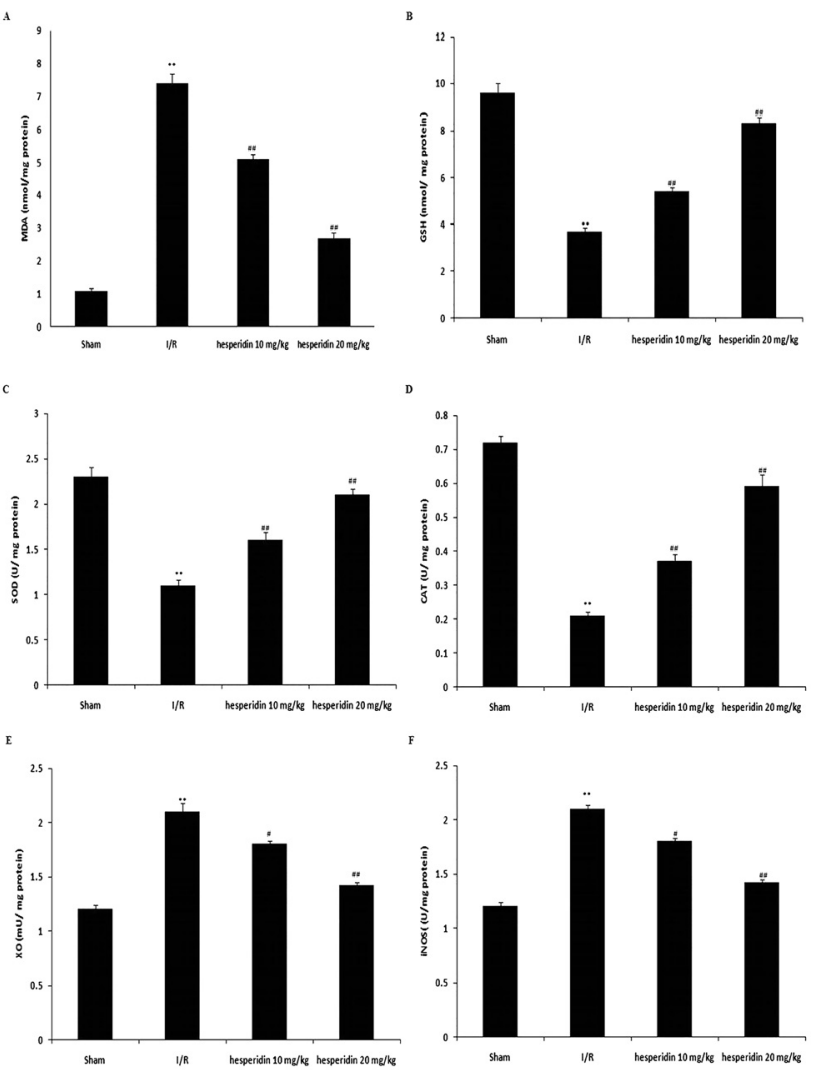

Figure 3. Hesperidin improves the altered parameters of oxidative stress in renal tissues from rats with $\mathrm{I} / \mathrm{R}-$ induced kidney injury. A: Concentration of MDA in the kidney tissue. B: Concentration of GSH in the kidney tissue. C: Activity of SOD in the kidney tissue. D: Activity of CAT in the kidney tissue. E: Activity of $\mathrm{XO}$ in the kidney tissue. F: Activity of iNOS in the kidney tissue. Data are presented as means \pm SEM $(n=$ 10). ${ }^{*} P<0.01$ compared to the control group; ${ }^{\# \#} P<0.01$ compared to the I/R group
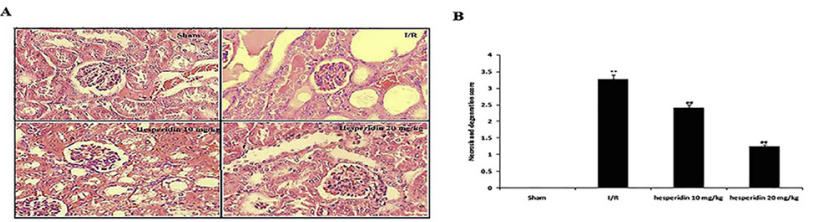

Figure 4. Hesperidin ameliorates the histopathological changes in renal tissues of rats with I/R-induced kidney injury. A: Histopathology of renal tissues as indicated by H\&E staining $(100 \times)$. B: Necrosis and degeneration scores. Data are presented as means \pm SEM $(n=10) .{ }^{*} P<0.01$ compared to the control group; ${ }^{\# \#} P<0.01$ compared to the I/R group 




Figure 5. Hesperidin ameliorates the altered expression TLR-4, NF- $\kappa \mathrm{B}, \mathrm{p}-\mathrm{I} \kappa \mathrm{B} \alpha$, and caspase- 3 protein in the renal tissues of rats with I/R-induced kidney injury. A: Western blots. B: Expression levels of TLR-4, NF- $\kappa \mathrm{B}, \mathrm{p}-\mathrm{I} \kappa \mathrm{B} \alpha$, and caspase- 3 protein. Data are presented as means $\pm \operatorname{SEM}(n=10) .{ }^{*} P<0.01$ compared to the control group; ${ }^{\# \#} P<0.01$ compared to the I/R group

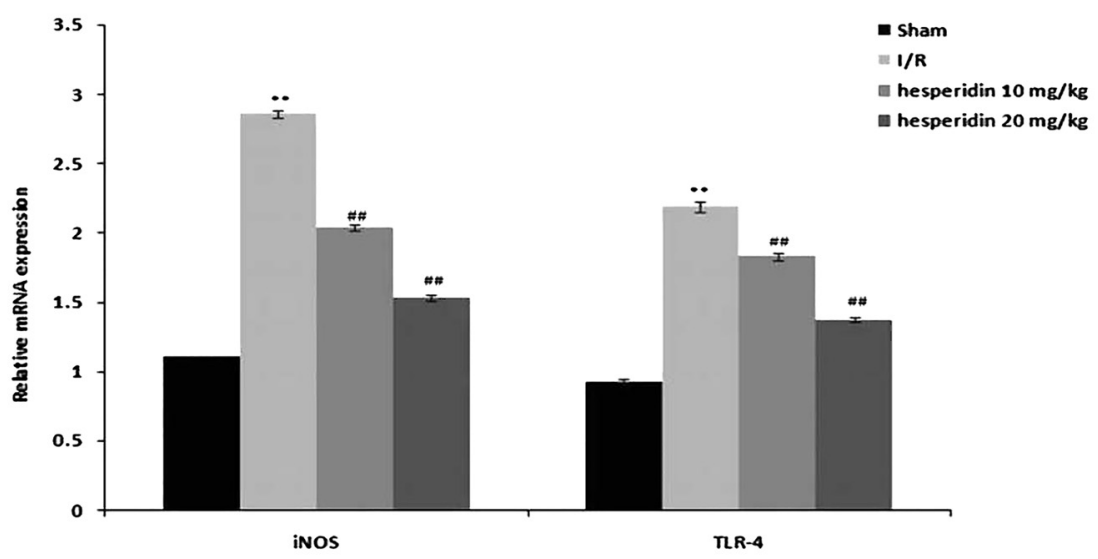

Figure 6. Hesperidin improves the altered mRNA expression TLR-4 and iNOS in the renal tissues of rats with I/R-induced kidney injury. Data are presented as means $\pm \operatorname{SEM}(n=10) .{ }^{* *} P<0.01$ compared to the control group; ${ }^{\# \#} P<0.01$ compared to the I/R group

injury (Fig. 6). There was an increase in the mRNA expression of TLR-4 and iNOS in the renal tissues of the I/R group compared to the control group. However, treatment with hesperidin decreased the mRNA expression of TLR-4 and iNOS.

\section{DISCUSSION}

Renal injury is reported to have a high mortality rate, and there are several limitations to current conventional treatments that are used to manage it [20]. This report evaluated the protective effect of hesperidin against I/R-induced kidney injury in rats. The effect of hesperidin on renal function, serum inflammatory mediators, and the level of oxidative stress in renal tissues was observed in rats with I/R-induced kidney injury. Moreover, protein expression and mRNA expression in the kidney tissues were determined by Western blotting and RT-PCR, respectively. Kidney tissues were analyzed using H\&E staining for histopathological changes. 
There are several mechanisms of pathogenesis that are involved in renal injury. One example is $I / R$, because it disturbs the balance between the antioxidant system and the production of reactive oxygen species [6]. Oxidative stress leads to cell death via the mitochondrial pathway, which includes the necrosis and apoptosis pathways [17]. Oxidative stress affects molecules such as MDA and GSH, while also altering the activity of several enzymes, including CAT, SOD, XO, and iNOS [13]. Drugs used for the management of kidney disease are reported to have strong antioxidant activity [19]. Hesperidin is a natural substance that is reported to possess strong antioxidant activity [22]. This study also revealed that hesperidin attenuated the enhanced oxidative stress in the renal tissues of rats with I/R-induced kidney injury.

Inflammation is a major factor that contributes to the development of renal injury. Moreover, inflammatory mediators like TNF- $\alpha$ and IL- $1 \beta$ are involved in renal injury [4]. It is well documented that oxidative stress leads to enhanced production of TNF- $\alpha$ and IL- $1 \beta$, which further contributes to kidney injury [15]. IL-10 has also been reported to be an anti-inflammatory mediator that protects against renal injury [7]. Our data suggest that treatment with hesperidin significantly reduced the levels of TNF- $\alpha$ and IL- $1 \beta$ while increasing the serum level of IL-10 compared to rats with I/R-induced kidney injury.

TLR-4 activates the immune system at the site of injured tissues. TLR- 4 has also been reported to initiate the production of intrarenal mediators of inflammation. Moreover, TLR-4 enhances the levels of TNF- $\alpha$ and IL-1 $\beta$ in renal injury [23]. The activation of oxidative stress and NF- $\kappa \mathrm{B}$ regulates the further downstream production of cytokines, which contribute to the development of renal injury [5]. Other investigations have also found that $\mathrm{I} / \mathrm{R}$ induces renal injury by altering the TLR-4/NF- $\kappa \mathrm{B}$ signaling pathway [18]. Our study shows that treatment with hesperidin attenuated the alteration of the TLR-4/NF- $\kappa \mathrm{B}$ signaling pathway in renal tissues of rats with I/R-induced kidney injury. Moreover, histopathological analysis revealed that I/R induces kidney injury by activating apoptosis. Apoptosis is activated by enhancing the activity of caspase- 3 in a downstream cascade. Data from our investigation suggest that hesperidin ameliorates apoptosis given that it reduces the activity of caspase- 3 in the renal tissues of rats with $\mathrm{I} /$ R-induced renal injury.

\section{CONCLUSION}

Hesperidin protected against renal injury in rats with I/R-induced kidney injury. It also reduced the level of inflammatory mediators and oxidative stress in I/R-induced kidney injury and thereby increased TLR-4/NF- $\kappa$ B/iNOS signaling.

Conflicts of interest: The authors declare that they have no conflicts of interest to disclose.

\section{ACKNOWLEDGMENTS}

The authors thank the Affiliated Hospital of Jining Medical University (Jining, China) for providing the necessary facilities to conduct the presented work. 


\section{REFERENCES}

1. Abuelsaad AS, Mohamed I, Allam G, Al-Solumani AA. Antimicrobial and immunomodulating activities of hesperidin and ellagic acid against diarrheic Aeromonas hydrophila in a murine model. Life Sci 2013; 93(20): 714-22, https://doi.org/10.1016/j.lfs.2013.09.019.

2. Arsenijevic J, Pavlova M, Rechel B, Groot W. Catastrophic health care expenditure among older people with chronic diseases in 15 European countries. PLoS One 2016; 11(7): e0157765, https://doi.org/10.1371/journal. pone.0157765.

3. Case J, Khan S, Khalid R, Khan A. Epidemiology of acute kidney injury in the intensive care unit. Crit Care Res Pract 2013; 2013: 479730, https://doi.org/10.1155/2013/479730.

4. Chen L, Deng H, Cui H, Fang J, Zuo Z, Deng J, et al. Inflammatory responses and inflammation-associated diseases in organs. Oncotarget 2017; 9(6): 7204-18, https://doi.org/10.18632/oncotarget.23208.

5. Chenxu G, Minxuan X, Yuting Q, Tingting G, Jinxiao L, Mingxing W, et al. iRhom2 loss alleviates renal injury in long-term PM2.5-exposed mice by suppression of inflammation and oxidative stress. Redox Biol 2018; 19: 147-57, https://doi.org/10.1016/j.redox.2018.08.009.

6. Dennis JM, Witting PK. Protective role for antioxidants in acute kidney disease. Nutrients 2017; 9(7): 718, https://doi.org/10.3390/nu9070718.

7. Du T, Zhu YJ. The regulation of inflammatory mediators in acute kidney injury via exogenous mesenchymal stem cells. Mediators Inflamm 2014; 2014: 261697, https://doi.org/10.1155/2014/261697.

8. Hsu CY, Ordoñez JD, Chertow GM, Fan D, McCulloch CE, Go AS. The risk of acute renal failure in patients with chronic kidney disease. Kidney Int 2008; 74(1): 101-7, https://doi.org/10.1038/ki.2008.107.

9. Kumar S, Pandey AK. Chemistry and biological activities of flavonoids: an overview. Sci World J 2013; 2013 : 162750, https://doi.org/10.1155/2013/162750.

10. Lee K-A, Lee S-H, Lee Y-J, Baeg SM, Shim J-H. Hesperidin induces apoptosis by inhibiting Sp1 and its regulatory protein in MSTO-211H cells. Biomol Ther (Seoul) 2012; 20(3): 273-9, https://doi.org/10.4062/ biomolther.2012.20.3.273.

11. Li M, Guo Z, Shao H, Qin B. Therapeutic effect of hesperidin on severe acute pancreatitis in rats and its mechanism. Zhonghua Wei Zhong Bing Ji Jiu Yi Xue 2017; 29(10): 921-5, https://doi.org/10.3760/cma.j.issn. 2095-4352.2017.10.012.

12. Malek M, Nematbakhsh M. Renal ischemia/reperfusion injury; from pathophysiology to treatment. J Renal Inj Prev 2015; 4(2): 20-7, https://doi.org/10.12861/jrip.2015.06.

13. Marrocco I, Altieri F, Peluso I. Measurement and clinical significance of biomarkers of oxidative stress in humans. Oxid Med Cell Longev 2017; 2017: 6501046, https://doi.org/10.1155/2017/6501046.

14. Milenkovic D, Deval C, Dubray C, Mazur A, Morand C. Hesperidin displays relevant role in the nutrigenomic effect of orange juice on blood leukocytes in human volunteers: a randomized controlled cross-over study. PLoS One 2011; 6(11): e26669, https://doi.org/10.1371/journal.pone.0026669.

15. Mittal M, Siddiqui MR, Tran K, Reddy SP, Malik AB. Reactive oxygen species in inflammation and tissue injury. Antioxid Redox Signal 2014; 20(7): 1126-67, https://doi.org/10.1089/ars.2012.5149.

16. Parhiz H, Roohbakhsh A, Soltani F, Rezaee R, Iranshahi M. Antioxidant and anti-inflammatory properties of the citrus flavonoids hesperidin and hesperetin: an updated review of their molecular mechanisms and experimental models. Phytother Res 2015; 29(3): 323-31, https://doi.org/10.1002/ptr.5256.

17. Ryter SW, Kim HP, Hoetzel A, Park JW, Nakahira K, Wang X, et al. Mechanisms of cell death in oxidative stress. Antioxid Redox Signal 2007; 9(1): 49-89, https://doi.org/10.1089/ars.2007.9.49. 
18. Sherif IO, Al-Shaalan NH. Vildagliptin attenuates hepatic ischemia/reperfusion injury via the TLR4/NF- $\kappa \mathrm{B}$ signaling pathway. Oxid Med Cell Longev 2018; 2018: 3509091, https://doi.org/10.1155/2018/3509091.

19. Tripathi AS, Mazumder PM, Chandewar AV. Sildenafil, a phosphodiesterase type 5 inhibitor, attenuates diabetic nephropathy in STZ-induced diabetic rats. J Basic Clin Physiol Pharmacol 2016; 27(1): 57-62, https:// doi.org/10.1515/jbcpp-2015-0035.

20. Vaidya VS, Ferguson MA, Bonventre JV: Biomarkers of acute kidney injury. Annu Rev Pharmacol Toxicol 2008; 48: 463-93, https://doi.org/10.1146/annurev.pharmtox.48.113006.094615.

21. Xia R, Sheng X, Xu X, Yu C, Lu H. Hesperidin induces apoptosis and G0/G1 arrest in human non-small cell lung cancer A549 cells. Int J Mol Med 2018; 41(1): 464-72, https://doi.org/10.3892/ijmm.2017.3250.

22. Yamamoto M, Jokura H, Hashizume K, Ominami H, Shibuya Y, Suzuki A, et al. Hesperidin metabolite hesperetin-7-O-glucuronide, but not hesperetin-3'-O-glucuronide, exerts hypotensive, vasodilatory, and antiinflammatory activities. Food Funct 2013; 4(9): 1346-51, https://doi.org/10.1039/c3fo60030k.

23. Zhang B, Ramesh G, Uematsu S, Akira S, Reeves WB. TLR4 signaling mediates inflammation and tissue injury in nephrotoxicity. J Am Soc Nephrol 2008; 19(5): 923-32, https://doi.org/10.1681/ASN.2007090982. 